\title{
Distributed Optimal Charging of Electric Vehicles for Demand Response and Load Shaping
}

\author{
Caroline Le Floch ${ }^{\mathrm{a}}$, Francois Belletti ${ }^{\mathrm{b}}$, Samveg Saxena ${ }^{\mathrm{c}}$, Alexandre M. Bayen ${ }^{\mathrm{a}, \mathrm{b}}$, Scott Moura ${ }^{\mathrm{a}}$
}

\begin{abstract}
This paper proposes three novel distributed algorithms to optimally schedule Plug-in Electric Vehicle (PEV) charging. We first define the global optimization problem, where we seek to control large heterogeneous fleets of PEVs to flatten a net Load Curve. We demonstrate that the aggregated objective can be distributed, via a new consensus variable. This leads to a dual maximization problem that can be solved in an iterative and decentralized manner: at each iteration, PEVs solve their optimal problem, communicate their response to the aggregator, which then updates a price signal. We propose three distributed algorithms to compute the optimal solution, namely a gradient ascent and two incremental stochastic gradient methods. We prove their rate of convergence, their precision level and expose their characteristics in terms of communication and privacy. Finally, we use the Vehicle-To-Grid simulator (V2Gsim), and present a set of case studies, with an application to flattening the "Duck Curve" in California.
\end{abstract}

\section{INTRODUCTION}

PEVs provide a compelling opportunity for supplying demand-side management services in the smart grid. Namely, a vehicle-to-grid (V2G) capable PEV communicates with the grid, stores energy, and can return energy to the electric grid. If properly managed, PEVs can enhance energy infrastructure resilience, enable renewable integration, and reduce economic costs for consumers and energy providers [1]. In addition to these societal-level infrastructure and environmental benefits, V2G may provide additional revenue streams to PEV owners [2]. Underscoring this opportunity, U.S. personal vehicles are parked and un-used $96 \%$ of time [3]. A single PEV can generally provide 5-20 kW, which is insufficient to participate in power grid markets. However, populations of PEVs can be aggregated to collectively provide grid services [4]. The main challenge, however, is monitoring and managing a large population of distributed PEV resources without sacrificing their primary function of personal mobility.

A growing body of literature addresses design of smart charging algorithms for PEV control, and propose either centralized or distributed protocols. Centralized algorithms [5], [6], [7] require a central infrastructure to communicate with each agent, collect all the information and compute the optimal load profile of the fleet. However, when the number

This work was supported in part by the France-Berkeley Fund Project, 2014-0036

${ }^{a}$ Civil and Environmental Engineering, University of California, Berkeley, United States, caroline.le-flochaberkeley.edu

${ }^{b}$ Electrical Engineering and Computer Sciences, University of California, Berkeley, United States

${ }^{c}$ Environmental Energy Technologies Division, Lawrence Berkeley National Laboratory of agents grows, these methods require heavy communication, high memory and computation time.

On the other hand, in distributed optimization problems, each PEV solves a local problem and communicates in a peer to peer fashion. A number of recent studies have focused on these methods to address coordinated charging of PEVs for load shaping. Previous related work, e.g. [8], [9] addresses "valley filling" only, with the objective to fill the overnight valley of load. In these models, a PEV plugs-in during a long and known time period (e.g. overnight), and must be fully charged at the end of this period. This does not encompass more comprehensive PEV usage schedules and does not consider V2G capabilities. The authors in [10] propose a high security and privacy-preserving framework based on Regret Minimization methods, but this requires constant constraints from day to day. Reference [11] addresses a richer set of load shaping, and proposes a framework based on the Alternating Direction Method of Multipliers (ADMM) to solve several optimization objectives for heterogeneous PEV populations.

In this paper, we propose a novel dual splitting algorithm and variants, which adds to existing studies on ADMM, and is applied to the California energy "Duck Curve". The Duck Chart was established by the California Independent System Operator (CAISO) [12] as a possible future net load curve for California. Net load is the difference between forecasted load (consumption) and electricity production from renewables. High penetration of solar generation is expected to create a deep valley during the day and steep ramping between mid afternoon and beginning of evening, which will require more flexible resources to avoid high ramping and overgeneration. In this paper we study how PEVs can provide demand response to smooth this Duck Curve.

The main contributions of our work include the following:

- We derive a novel distributed algorithm based on dual splitting methods. This framework includes battery degradation costs and is applicable to the most general driver behavior, where PEVs can either be used in $G 2 \mathrm{~V}$ (can only consume energy) or $V 2 G$ mode (can consume and supply energy).

- We propose three different algorithms, namely a gradient ascent and two stochastic incremental methods. Contrary to the ADMM in [11], the proposed method exploits the independence between constraints, which has strong repercussions on time complexity and privacy preserving properties.

- We perform theoretical convergence analysis with explicit convergence bounds. In practice, this is essential to measure implementation burdens, and assess the cyber- 
security properties of the algorithm.

- We use real data from National Household Travel Survey (NHTS) [13] to quantify demand response opportunities in California for various scenarios of PEV penetration.

The remainder of the paper is organized as follows. Section IIIformulates the optimal scheduling problem. Section III establishes a dual splitting method, proposes three distributed algorithms and analyzes their convergence properties. Section IV applies the algorithm to real data in California.

TABLE I

NOMENCLATURE

\begin{tabular}{ll}
\hline \hline Symbol & Description \\
\hline$N$ & Number of PEVs \\
$T$ & Time horizon \\
$u_{n}^{t}$ & Charging rate of car $n$ at time $t$ \\
$c_{n}^{t}$ & Discharging rate of PEV $n$ at time $t$ due to driving \\
$x_{n}^{t}$ & State Of Charge (SOC) of PEV $n$ at time $t$ \\
$D^{t}$ & External loads at time $\mathrm{t}$ (consumption - solar generation) \\
$B_{n}$ & Battery capacity of PEV $\mathrm{n}$ \\
$x_{n}^{\min }$ & Minimum State Of Charge (SOC) of PEV $\mathrm{n}$ \\
$x_{n}^{\max }$ & Maximum State Of Charge (SOC) of PEV $\mathrm{n}$ \\
$P_{n}^{t}$ & Maximum charging power of PEV $\mathrm{n}$ at time $t$ \\
$P_{n}^{t}$ & Minimum charging power of PEV $\mathrm{n}$ at time $t$ \\
\hline \hline
\end{tabular}

\section{Problem FORMUlation}

In this section, we use the notation in Table I and develop an optimization program to design optimal PEV charging schedules. We use double brackets to denote a discrete set, e.g. $[[1, T]]=\{1,2, \cdots, T-1, T\}$.

\section{A. Electric Vehicle Charging Model}

For each vehicle, we consider linear battery dynamics. Let $x_{n}^{t}$ denote the State of Charge (SOC) of vehicle $n$ at time $t$, $u_{n}^{t}$ the charging rate when vehicle $n$ is plugged-in, and $c_{n}^{t}$ the driving discharging rate when vehicle $n$ is on road. The battery dynamics are described by a linear model, with a power conversion efficiency $\eta \leq 1$.

$$
\begin{aligned}
x_{n}^{t+1} & =x_{n}^{t}+\frac{\eta^{m} u_{n}^{t}}{B_{n}} \Delta t-\frac{c_{n}^{t}}{\eta B_{n}} \Delta t . \\
m & = \begin{cases}1 & \text { if } u_{n}^{t} \geq 0, \\
-1 & \text { if } u_{n}^{t}<0,\end{cases} \\
x_{n}^{\min } \leq \quad x_{n}^{t} & \leq x_{n}^{\max }
\end{aligned}
$$

\section{B. Charging constraints}

The variable $u_{n}^{t}$ can be non zero if and only if PEV $n$ is plugged-in at time $t$. We denote $R_{n}$ the indicator vector:

$$
R_{n}^{t}=\left\{\begin{array}{l}
1, \text { if PEV } n \text { is plugged in at time } \mathrm{t} \\
0, \text { otherwise }
\end{array}\right.
$$

From this definition, we can derive the equality constraint:

$$
\left(1-R_{n}\right)^{T} u_{n}=0
$$

Equations (1), (2) and (3) define a constraint set, which is more binding as $\eta$ increases, and attains the most binding case when $\eta=1$ (in practice, $\eta=1$ models a perfect battery efficiency). Therefore, satisfying the constraints associated with a perfect efficiency ensures that the constraints (1), (2), (3) are true at every time step $t \in \llbracket 1, T \rrbracket$, for any value of $\eta \leq 1$. For simplicity, and similar to previous work ([14], [15]), we will use $\eta=1$ to determine PEV energy storage and power constraints:

$$
\begin{gathered}
\frac{B_{n}}{\Delta t}\left(x_{n}^{\text {min }}-x_{n}^{\text {init }}\right)+\sum_{\tau=1}^{t} c_{n}^{\tau} \leq \sum_{\tau=1}^{t} u_{n}^{\tau} \leq \frac{B_{n}}{\Delta t}\left(x_{n}^{\text {max }}-x_{n}^{\text {init }}\right)+\sum_{\tau=1}^{t} c_{n}^{\tau}(6) \\
\underline{P_{n}^{t}} \leq u_{n}^{t} \leq \overline{P_{n}^{t}}
\end{gathered}
$$

\section{Finite Time Horizon constraints}

The above problem has a fixed time horizon $T$. In practice, no terminal constraint could cause total depletion of energy at the end of the period $T$. For simplicity, we impose that every PEV attains at least SOC $x_{n}^{\text {final }}$ at the end of the period.

$$
\sum_{\tau=1}^{T} u_{n}^{\tau} \geq \frac{B_{n}}{\Delta t}\left(x_{n}^{\text {final }}-x_{n}^{\text {init }}\right)+\sum_{\tau=1}^{T} c_{n}^{\tau}
$$

This is a conservative constraint, which can be improved in future formulations of the problem.

\section{Objective}

We denote $D^{t}$ the aggregate electric loads combined with the uncontrollable renewable generation. Variable $D^{t}$ is the net load and does not include PEV loads [12].

The objective of the control algorithm is to flatten the total load curve $D^{t}+\sum_{n=1}^{N} u_{n}^{t}$. We seek to minimize peaks and variance of the total energy needs. This can be formulated in the following objective:

$$
\min _{u} \sum_{t=1}^{T}\left(D^{t}+\sum_{n=1}^{N} u_{n}^{t}\right)^{2}
$$

The optimization program is a Quadratic Program with $T \times N$ linear equality constraints and $1+4 T \times N$ linear inequality constraints, summarized as follows:

$$
\begin{aligned}
& \min _{u} \sum_{t=1}^{T}\left(D^{t}+\sum_{n=1}^{N} u_{n}^{t}\right)^{2} \\
& \text { st } \forall n \in \llbracket 1, N \rrbracket, \forall t \in \llbracket 1, T \rrbracket \\
& \forall n \in \llbracket 1, N \rrbracket, \forall t \in \llbracket 1, T \rrbracket \\
& \left(1-R_{n}\right)^{T} u_{n}=0 \\
& \forall n \in \llbracket 1, N \rrbracket, \forall t \in \llbracket 1, T \rrbracket \\
& \underline{P_{n}^{t}} \leq u_{n}^{t} \leq \overline{P_{n}^{t}} \\
& \text { (6), (8) }
\end{aligned}
$$

\section{DuAl DECOMPOSITION}

In this section we develop a Dual splitting method and provide a distributed protocol to solve problem 10 . We show that, provided we add a regularization term, both problems are equivalent and the distributed protocol converges to the optimal solution. 


\section{A. Dual splitting}

In the remainder of this paper, we will study the regularized version of problem $(10)$. The objective function (9) is augmented with a penalization term $\sigma \sum_{n=1}^{N}\left\|u_{n}\right\|^{2}$. This $L_{2}$ norm term penalizes the sum of power squared and can be physically interpreted as a battery degradation penalty. Battery degradation depends on a multitude of factors, including solid electrolyte interphase film formation, electrolyte decomposition, particle fracture, electrode delamination, etc. [16]. Experimental aging studies [17] have shown that aging is highly correlated to the integral of power transferred through the battery. For this reason, we refer to the regularization term as the "Degradation cost" in the rest of this paper. Now, the problem can be rewritten in the compact form:

$$
\begin{array}{ll}
\min _{u} & \sum_{t=1}^{T}\left(D^{t}+\sum_{1}^{N} u_{n}^{t}\right)^{2}+\sigma \sum_{n=1}^{N}\left\|u_{n}\right\|^{2} \\
\text { st } & L_{n} \leq B u_{n} \leq M_{n} \forall n \\
& A_{n} u_{n}=0 \forall n
\end{array}
$$

We define the consensus variable $z^{t}=D^{t}+\sum_{n=1}^{N} u_{n}^{t}$; then the problem becomes:

$$
\begin{array}{cl}
\min _{u, z} & \sum_{t=1}^{T}\left(z^{t}\right)^{2}+\sigma \sum_{n=1}^{N}\left\|u_{n}\right\|^{2} \\
\text { st } \quad & z^{t}=D^{t}+\sum_{n=1}^{N} u_{n}^{t} \\
& L_{n} \leq B u_{n} \leq M_{n} \forall n \\
& A_{n} u_{n}=0 \forall n
\end{array}
$$

The above problem is a quadratic minimization problem with linear constraints, therefore it is convex. We can dualize the equality constraint $(12 b)$ and form the Lagrangian with dual variable $\lambda$. Moreover, we suppose there exists a feasible point $u$ in the convex set formed by the constraints $12 \mathrm{c}$ ) and (12d). Since (12b) is affine and always feasible, problem (12) is convex and admits a feasible point. Slater's condition holds [18] and strong duality gives the equivalent problem:

$$
\begin{array}{cl}
\max _{\lambda^{t} \in \Re} \min _{u, z} & \sum_{t=1}^{T}\left(z^{t}\right)^{2}+\sum_{t=1}^{T} \lambda^{t}\left(z^{t}-D^{t}-\sum_{n=1}^{N} u_{n}^{t}\right)+\sigma \sum_{n=1}^{N}\left\|u_{n}\right\|^{2} \\
\text { st } & L_{n} \leq B u_{n} \leq M_{n} \forall n . \\
& A_{n} u_{n}=0 \forall n .
\end{array}
$$

We first perform the minimization with respect to $z$ :

$$
\begin{aligned}
\forall t \in \llbracket 1, T \rrbracket z^{t^{*}} & =\underset{\operatorname{argmin}}{ }\left[f_{t}\left(z^{t}\right)=z^{t^{2}}+\lambda^{t} z^{t}\right] \\
z^{t^{*}} & =-\frac{\lambda^{t}}{2} \text { and } f_{t}\left(z^{t^{*}}\right)=-\frac{\left(\lambda^{t}\right)^{2}}{4}
\end{aligned}
$$

Now, we define $\mu^{t}=-\lambda^{t}$ and plug the value of $z^{t *}$ into problem 13. Then, the problem is equivalent to:

$$
\begin{aligned}
& \max _{\mu \in \Re^{T}} \frac{-\|\mu\|^{2}}{4}+\mu^{T} D+\sum_{n=1}^{N} \min _{u_{n}} \mu^{T} u_{n}+\sigma\left\|u_{n}\right\|^{2}(14 \mathrm{a}) \\
& \text { st } \quad L_{n} \leq B u_{n} \leq M_{n}(14 \mathrm{~b}) \\
& A_{n} u_{n}=0 \text {. }
\end{aligned}
$$

In the first term of (11), the PEV power is coupled by the cross terms $u_{n}^{t} \times u_{m}^{t}$ for all $n, m, t$. It is remarkable that the contributions of $u_{n}$ in the objective function $14 \mathrm{a}$ are decoupled along $n \in \llbracket 1, N \rrbracket$. Problem $[14$ can now be solved in a distributed manner. Unlike the ADMM, the approach above has leveraged the independence between PEV constraints. In the next sections we will show that this allows us to perform a precise convergence rate analysis, and study the performance of the Gradient Ascent Method and the Incremental Stochastic Gradient Method.

In the remainder of this paper, $\Omega_{n}$ denotes the non-empty convex set given by $14 b$, $14 c$.

\section{B. Gradient ascent method}

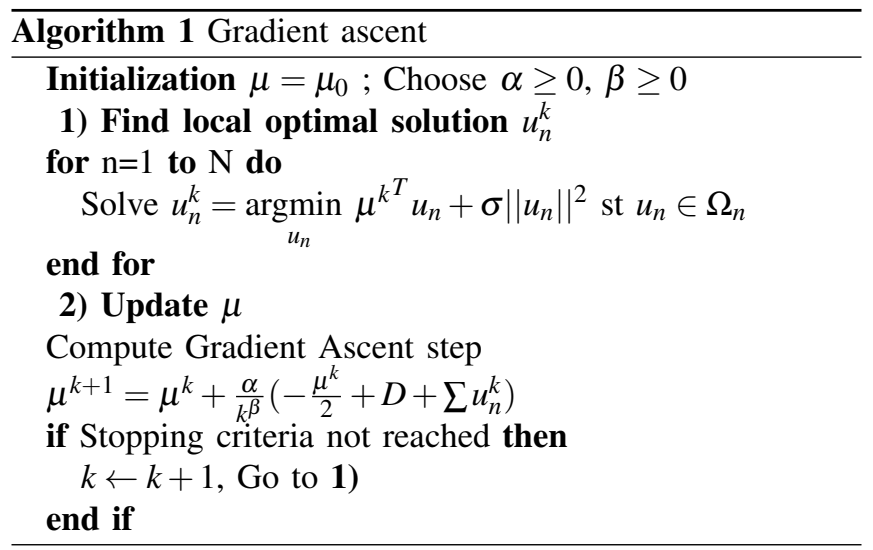

Algorithm 1 gives the gradient ascent protocol to solve the optimization program. In this section, we prove that Algorithm 1 converges to the optimal solution and we give complexity bounds. Let $g: \mathfrak{R}^{T} \rightarrow \mathfrak{R}$ denote the dual function:

$$
\begin{gathered}
g(\mu)=\frac{-\|\mu\|^{2}}{4}+\mu^{T} D+\sum_{n=1}^{N} \min _{u_{n}}\left(\mu^{T} u_{n}+\sigma\left\|u_{n}\right\|^{2}\right) \\
\text { st } u_{n} \in \Omega_{n}
\end{gathered}
$$

Theorem 1 (Gradient Ascent with constant step-size): The dual problem (14) has a unique solution $\mu^{*}$ and the gradient ascent with step-size $\alpha=\frac{2 \sigma}{\sigma+N}$ converges with

$$
g\left(\mu^{*}\right)-g\left(\mu_{k}\right) \leq\left(\frac{N}{\sigma+N}\right)^{k}\left(g(\mu *)-g\left(\mu_{0}\right)\right)
$$

Proof: We will prove Theorem 11 in two steps: first show strong concavity, then show that the function admits Lipschitz gradients.

Step 1: Show the function $g: \Re^{T} \rightarrow \Re$ is strongly concave with constant $m=\frac{1}{2}$. 
We refer to [18] for generic results about convex functions. Function $g$ is a sum of a strongly concave quadratic function and $N$ functions $\psi_{n}$ defined by:

$$
\begin{array}{cl}
\psi_{n}(\mu)=\min _{u_{n}} & \mu^{T} u_{n}+\sigma\left\|u_{n}\right\|^{2} \\
\text { st } & u_{n} \in \Omega_{n} .
\end{array}
$$

$\Omega_{n}$ is a non-empty convex set. For each $\mu, \psi_{n}(\mu)$ appears as a minimum of a strongly convex function over a convex set, thus it has a unique solution $u_{n}^{*}(\mu)$. Let $\tau \in[0,1], \mu_{1}, \mu_{2} \in \mathfrak{R}$.

$$
\begin{aligned}
& \tau \psi_{n}\left(\mu_{1}\right)+(1-\tau) \psi_{n}\left(\mu_{2}\right) \\
& =\tau \min _{u_{n} \in \Omega_{n}} \mu_{1}^{T} u_{n}+\sigma\left\|u_{n}\right\|^{2}+(1-\tau) \min _{u_{n} \in \Omega_{n}} \mu_{2}^{T} u_{n}+\sigma\left\|u_{n}\right\|^{2} \\
& \leq \min _{u_{n} \in \Omega_{n}} \tau\left(\mu_{1}^{T} u_{n}+\sigma\left\|u_{n}\right\|^{2}\right)+(1-\tau)\left(\mu_{2}^{T} u_{n}+\sigma\left\|u_{n}\right\|^{2}\right) \\
& =\psi_{n}\left(\tau \mu_{1}+(1-\tau) \mu_{2}\right)
\end{aligned}
$$

Therefore, $\psi_{n}$ is concave. Now, $g(\mu)=\frac{-\|\mu\|^{2}}{4}+\mu^{T} D+$ $\sum_{n=1}^{N} \psi_{n}$. The quadratic part is strongly concave with constant $\frac{1}{2}$, therefore $\mathrm{g}$ is at least $\frac{1}{2}$ strongly concave .

Step 2: Show the function $g: \Re^{T} \rightarrow \Re$ admits Lipschitz gradients with constant $L_{g}=\frac{1}{2}\left(1+\frac{N}{\sigma}\right)$.

Because $\psi_{n}(\mu)$ admits a unique minimum and the function is linear in $\mu, \psi_{n}$ is differentiable and $\nabla \psi_{n}(\mu)=u_{n}^{*}(\mu)$ (see [19]). Using the characterization of minimum of convex functions with $u_{n}^{* 1}=u_{n}^{*}\left(\mu_{1}\right)$ and $u_{n}^{* 2}=u_{n}^{*}\left(\mu_{2}\right)$, we have:

$$
\begin{aligned}
& \left\langle\mu_{1}+2 \sigma u_{n}^{* 1}, u_{n}-u_{n}^{* 1}\right\rangle \geq 0 \forall u_{n} \in \Omega_{n} \\
& \left\langle\mu_{2}+2 \sigma u_{n}^{* 2}, u_{n}-u_{n}^{* 2}\right\rangle \geq 0 \forall u_{n} \in \Omega_{n}
\end{aligned}
$$

Applying these relations to $u_{n}^{* 2}$ and $u_{n}^{* 1}$ respectively we get:

$$
\begin{aligned}
& \left\langle\mu_{1}+2 \sigma u_{n}^{* 1}, u_{n}^{* 2}-u_{n}^{* 1}\right\rangle \geq 0 \\
& \left\langle\mu_{2}+2 \sigma u_{n}^{* 2}, u_{n}^{* 1}-u_{n}^{* 2}\right\rangle \geq 0
\end{aligned}
$$

Adding these lines, and using Cauchy Schwarz yields :

$$
\begin{gathered}
\left\langle\left(\mu_{1}-\mu_{2}\right)+2 \sigma\left(u_{n}^{* 1}-u_{n}^{* 2}\right), u_{n}^{* 2}-u_{n}^{* 1}\right\rangle \geq 0 \\
\left\langle\left(\mu_{1}-\mu_{2}\right), u_{n}^{* 1}-u_{n}^{* 2}\right\rangle-2 \sigma\left\|u_{n}^{* 1}-u_{n}^{* 2}\right\|^{2} \geq 0 \\
\left\|\mu_{1}-\mu_{2}\left|\left\||| u_{n}^{* 2}-u_{n}^{* 1}\right\| \geq 2 \sigma\left\|u_{n}^{* 1}-u_{n}^{* 2}\right\|^{2}\right.\right.
\end{gathered}
$$

We conclude $\left\|u_{n}^{* 1}-u_{n}^{* 2}\right\| \leq \frac{1}{2 \sigma}\left\|\mu_{1}-\mu_{2}\right\|$.

$$
\begin{aligned}
\nabla g\left(\mu_{1}\right)-\nabla g\left(\mu_{2}\right) & =-\frac{\mu_{1}-\mu_{2}}{2}+\sum_{n}\left(u_{n}^{* 1}-u_{n}^{* 2}\right) \\
\left\|\nabla g\left(\mu_{1}\right)-\nabla g\left(\mu_{2}\right)\right\| & \leq \frac{\sigma+N}{2 \sigma}\left\|\mu_{1}-\mu_{2}\right\|
\end{aligned}
$$

Therefore, $g$ has Lipschitz gradients with constant $L_{g}=\frac{\sigma+N}{2 \sigma}$.

Now, from[18, Ch. 9, p. 466], the gradient ascent method with stepsize $\frac{1}{L_{g}}$ converges and gives

$$
\begin{aligned}
g\left(\mu^{*}\right)-g\left(\mu_{k}\right) & \leq\left(1-\frac{m}{L_{g}}\right)^{k}\left(g(\mu *)-g\left(\mu_{0}\right)\right) \\
& \leq\left(\frac{N}{\sigma+N}\right)^{k}\left(g(\mu *)-g\left(\mu_{0}\right)\right)
\end{aligned}
$$
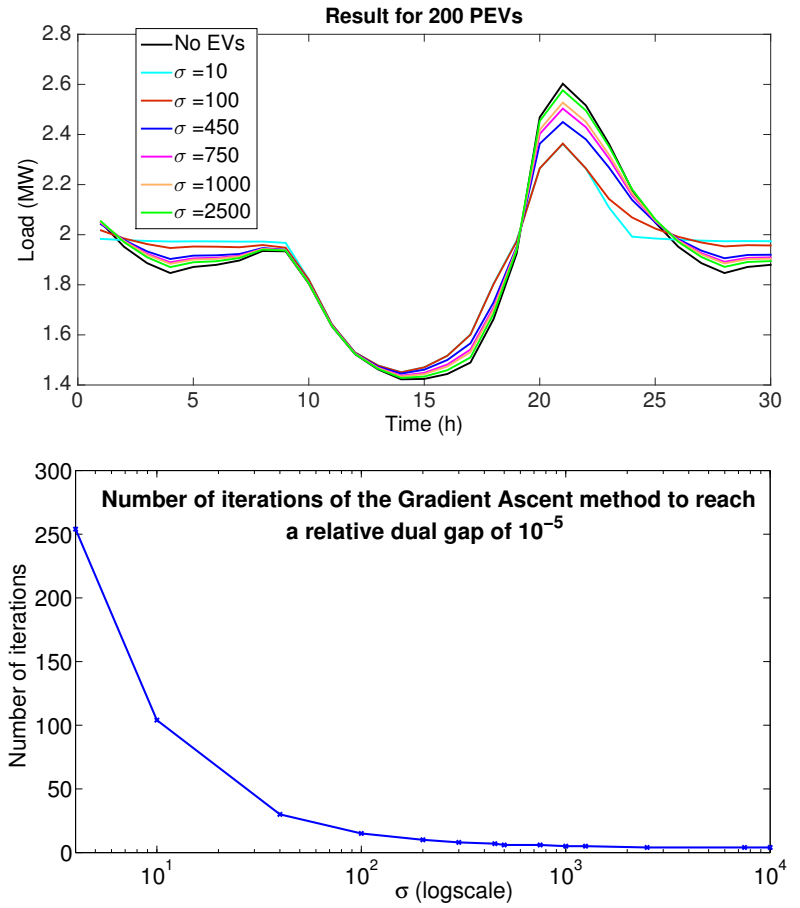

Fig. 1. Impact of $\sigma$ on convergence rate and results

Remark 1: Algorithm 1] with constant step-size converges to accuracy $\varepsilon$ in $O\left(\left(1+\frac{N}{\sigma}\right) \log \left(\frac{1}{\varepsilon}\right)\right)$ iterations, and complexity $O\left(\left(N+\frac{N^{2}}{\sigma}\right) \log \left(\frac{1}{\varepsilon}\right)\right)$. Hence, it is important to tune the parameter $\frac{\sigma}{N}$ to accelerate the convergence. On the other hand, $\frac{\sigma}{N}$ measures how selfish the agents are: as $\frac{\sigma}{N}$ increases, the penalization for battery degradation increases and the result looses optimality in terms of variance minimization. Figure 1 illustrates this tradeoff for 200 agents with $\mu_{0}=D$, where $D$ is the initial load curve. We stop the algorithm when we reach a relative duality gap of $10^{-5}$. We note that for $\frac{\sigma}{N} \geq 1,10$ iterations are enough to reach this precision.

Remark 2: The above derivations and proof remain true for any feasible convex set of constraints $\Omega_{n}$. Then, the algorithm can be adapted to any similar problem where each agent has an independent set of convex constraints. In particular, this derivation remains true when we want to study uncertainties and introduce a robust convex set of constraints.

\section{Incremental Stochastic Gradient Method}

In this section, we show that we can use an Incremental Stochastic Gradient Method (ISGM) to solve problem (14a). We keep the same notations and remark that $\mathrm{g}$ can be expressed as:

$$
\begin{array}{rlrl}
g(\mu) & =\frac{1}{N} \sum_{n=1}^{N} \frac{-\|\mu\|^{2}}{4}+\mu^{T} D+ & N \min _{u_{n}} \mu^{T} u_{n}+\sigma\left\|u_{n}\right\|^{2} \\
\text { st } u_{n} \in \Omega_{n} & \\
& =\frac{1}{N} \sum_{n=1}^{N} g_{n}(\mu)
\end{array}
$$

The incremental gradient method is a version of Stochastic Gradient Method where we pick $i \in \llbracket 1, N \rrbracket$ uniformly at random, and choose the iterate direction $\nabla g_{i}$. We note $\mu^{*}$ 


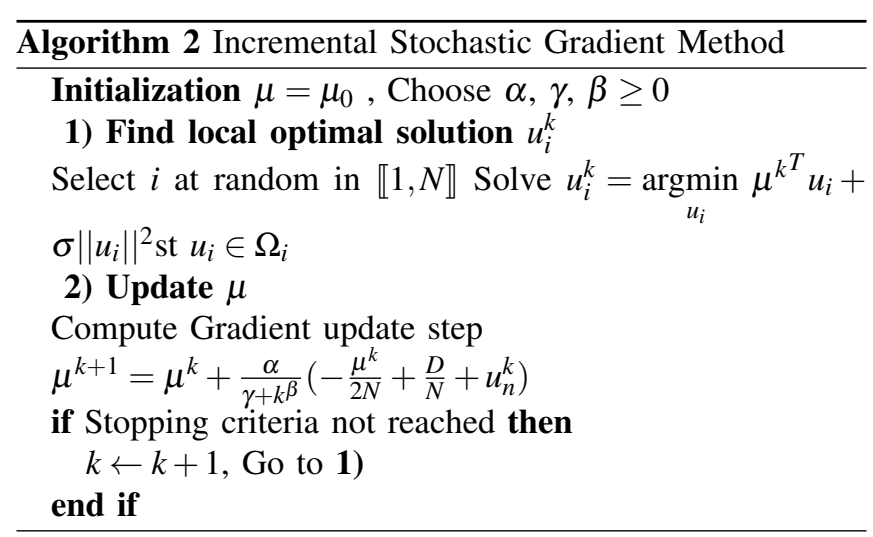

the optimum for $g$ and $\mu_{n}^{*}$ the optimum for $g_{n}$.

Theorem 2 (ISGM with constant step-size): ISGM with constant step-size $\alpha \in\left[0, \frac{1}{(1+N / \sigma)^{2}}\right]$ reaches the ball $B\left(\mu^{*}, r\right)$ with precision $\varepsilon$ where $r=\frac{1}{1+2 \alpha L_{g}^{2}} \frac{2 \alpha}{N} L_{g}^{3} \sum_{i=1}^{N}\left\|\mu_{i}^{*}-\mu^{*}\right\|^{2}$ in $\frac{1}{\alpha\left(1-2 \alpha L_{g}^{2}\right)} \ln \left(\frac{\left\|\mu_{0}-\mu^{*}\right\|}{\varepsilon}\right)$ iterations.

Theorem 3 (ISGM with decreasing step-size): ISGM with decreasing step-size $\alpha_{k}=\frac{1}{(1+N / \sigma)^{2}+k}$ converges to the optimal solution $\mu^{*}$ and

$\mathbb{E}\left(g\left(\mu^{*}\right)-g\left(\mu_{k}\right)\right) \leq \frac{1}{N} \sum_{i=1}^{N}\left\|\mu_{i}^{*}-\mu^{*}\right\|^{2} \frac{1}{(1+N / \sigma)^{2}+k}$

Proof of Theorems: We prove Theorem 2 and 3 by showing that we can find $L$ and $B$ such that $\mathbb{E}\left(\|\nabla g(\mu)\|^{2}\right) \leq L^{2} \| \mu-$ $\mu^{*} \|^{2}+B^{2}$.

Step 1: Show the function $g_{n}: \Re^{T} \rightarrow \Re$ admits Lipschitz gradients with constant $L_{n}=L_{g}=\frac{1}{2}\left(1+\frac{N}{\sigma}\right)$.

This is shown by following the same proof as step 2 of Theorem 1 .

Step 2: Show $\mathbb{E}\left(\left\|\nabla g_{i}(\mu)\right\|^{2}\right) \leq 2 L_{g}^{2}\left\|\mu-\mu^{*}\right\|^{2}+B^{2}$ with $L_{g}=\frac{1}{2}\left(1+\frac{N}{\sigma}\right)$ and $B^{2}=\frac{1}{2 N}\left(1+\frac{N}{\sigma}\right)^{2} \sum_{i=1}^{N}\left\|\mu_{i}^{*}-\mu^{*}\right\|^{2}$. Using Cauchy Schwarz inequality and the Lispchitz condition, we obtain:

$$
\begin{aligned}
\mathbb{E}\left(\left\|\nabla g_{i}(\mu)\right\|^{2}\right) & \leq \mathbb{E}\left(L_{i}^{2}\left\|\mu-\mu_{i}^{*}\right\|^{2}\right) \\
& \leq \mathbb{E}\left(2 L_{i}^{2}\left\|\mu-\mu^{*}\right\|^{2}+2 L_{i}^{2}\left\|\mu_{i}^{*}-\mu^{*}\right\|^{2}\right) \\
& =\frac{2}{N} \sum_{i=1}^{N} L_{g}^{2}\left\|\mu-\mu^{*}\right\|^{2}+\frac{2}{N} \sum_{i=1}^{N} L_{g}^{2}\left\|\mu_{i}^{*}-\mu^{*}\right\|^{2} \\
& =2 L_{g}^{2}\left\|\mu-\mu^{*}\right\|^{2}+B^{2}
\end{aligned}
$$

This is the condition $\mathbb{E}\left(\|\nabla g(\mu)\|^{2}\right) \leq L^{2}\left\|\mu-\mu^{*}\right\|^{2}+B^{2}$. With these particular values of $L$ and $B$, results in [20] can be used to establish the step-sizes and convergence rates of Theorem 2 and 3 .

Remark 3: The convergence rates of Algorithm 1] and Algorithm 2 with constant step size are linear, whereas the convergence rate of Algorithm 2 with decreasing step size is as $\frac{1}{k}$, which is much slower. An Incremental Method iteration involves only one agent and is $\mathrm{N}$ times faster than a Gradient Ascent iteration. Thus the convergence speed of Algorithm 2

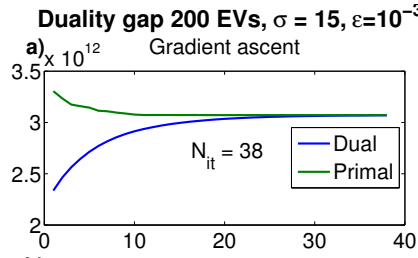

b) $\times 10^{12}$ Incremental - constant stepsize

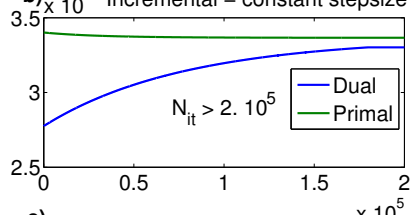

c) $\times 10^{12}$ Incremental - decreasing stepsize
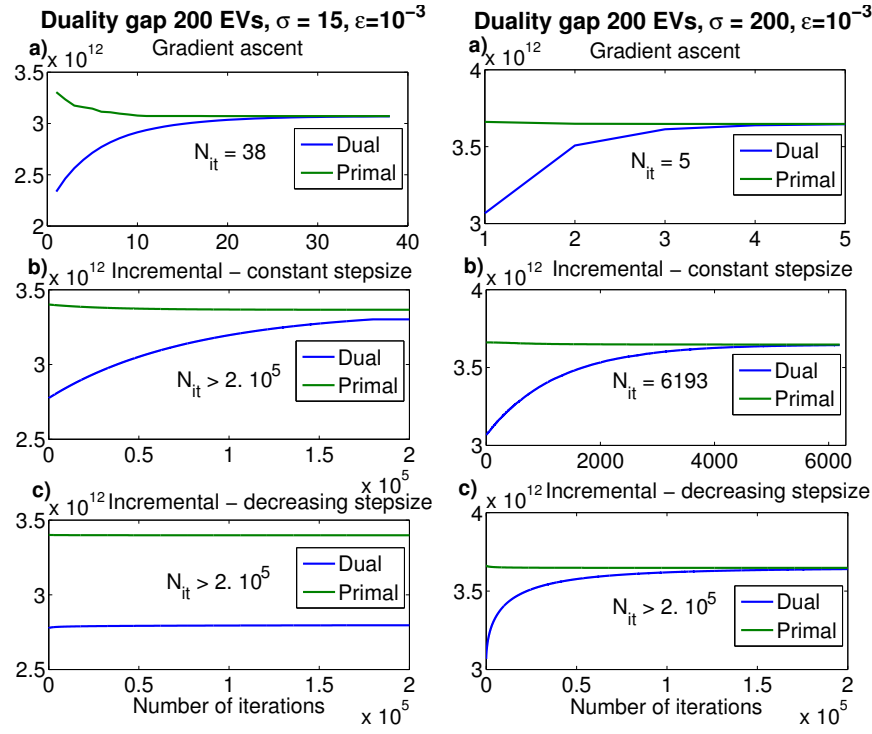

Fig. 2. Duality gap $\sigma=15$

Fig. 3. Duality gap $\sigma=200$

with constant step-size is usually faster, but converges only to a certain precision $r$. This algorithm should be used when the aggregator needs a fast convergence and is satisfied with an approximate solution. In this stochastic configuration, only one random PEV at a time needs to communicate with the aggregator. This usually reduces the amount of messages that one agent needs to send to the aggregator, improves the resistance to hacking attacks [21], and has better cybersecurity performance (see a companion paper [22], which specifically addresses the privacy preserving properties of the algorithm). Thus, Algorithm 2 with decreasing step-size should be used when the aggregator is concerned about cyber security. Figure 2 and 3 show the values of the primal and dual objectives of the function for each of the three methods. We stop Algorithm 1 and 2 when the number of iteration exceeds $2 \times 10^{5}$ or the relative duality gap reaches $10^{-3} ; N_{i t}$ denotes the number of iterations to converge to the precision $\varepsilon=10^{-3}$ with the starting point $\mu_{0}=D$, where $D$ is the load curve ("Duck Curve"). This shows that all the algorithms converge faster as the parameter $\frac{\sigma}{N}$ increases. For $\sigma=200$, Algorithm 1 and Algorithm 2 with constant step-size converge to the required precision:

- Algorithm 1 needs 5 full-gradient iterations.

- Algorithm 2 needs 6193 stochastic iterations, which corresponds to $\frac{6193}{200} \simeq 31$ full-gradient iterations.

\section{Results, application to the Duck Curve}

In this section, we use the Vehicle-to-Grid Simulator (V2G-Sim) developed by the Grid Integration Group at Lawrence Berkeley National Laboratory [23]. V2G-Sim is an agent-based simulator that models the driving and charging behavior of individual PEVs and their grid impact. Reference [24] gives more details about the V2G-Sim methodology.

\section{A. Impact of charging infrastructure}

NHTS gives a choice of representative location types for trip departures and arrivals (eg: Home, Work, Restaurant, 
TABLE II

DESCRIPTION OF CASE STUDIES

\begin{tabular}{|l|cc|cc|cc|}
\hline \multicolumn{1}{|c|}{ Case Study } & \multicolumn{2}{|c|}{ Home } & \multicolumn{2}{c|}{ Work } & \multicolumn{2}{c|}{ Other } \\
& L1 & L2 & L1 & L2 & L1 & L2 \\
\hline L1 Home & $100 \%$ & 0 & 0 & 0 & 0 & 0 \\
L1 Home, Work & $100 \%$ & 0 & $100 \%$ & 0 & 0 & 0 \\
Mix & $50 \%$ & $50 \%$ & $20 \%$ & $20 \%$ & $15 \%$ & $5 \%$ \\
\hline
\end{tabular}
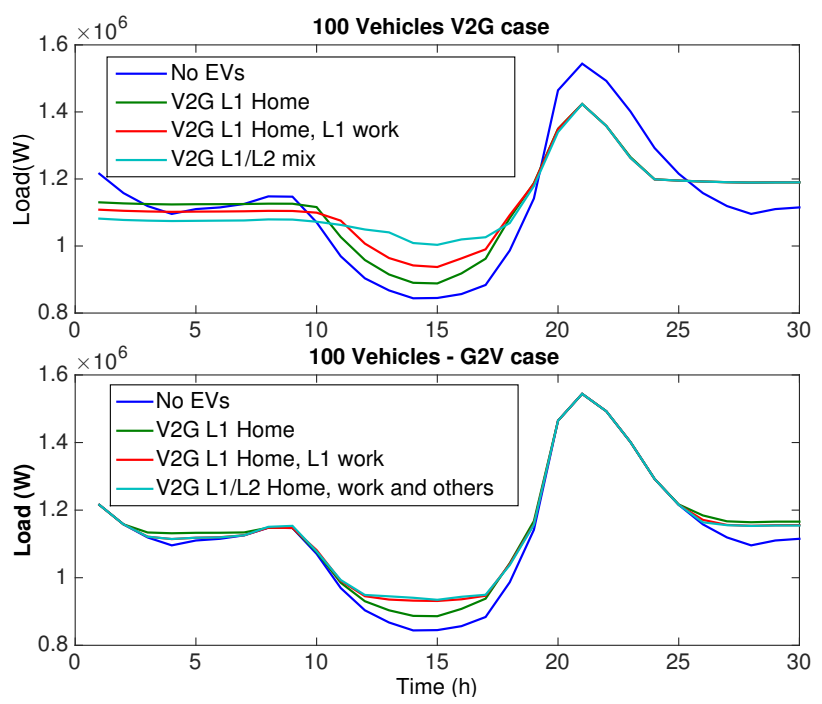

Fig. 4. Impact of the charging infrastructure

School/Church, Medical/Dental). Based on this information, we simulate different infrastructure levels and generate corresponding charging constraints for the algorithm. Level 1 (L1) chargers correspond to $\bar{P}=1400 \mathrm{~W}$, and Level 2 (L2) chargers correspond to $\bar{P}=7200 \mathrm{~W}$. We study two different cases: in the $V 2 G$ case PEVs can either charge or supply energy when they are plugged-in, and in the $G 2 V$ case PEVs can only charge. Table $\Pi$ presents 3 different infrastructure scenarios and Fig. 4 shows how it impacts the loads for 100 PEVs, depending on their V2G capability. This case study shows that the charging infrastructure significantly impacts the demand response opportunities of PEVs. In particular, L2 chargers with V2G capabilities facilitate peak reduction.

\section{B. Impact of PEV penetration}

In this section, we fix the scale $\frac{\text { Number of cars }}{\text { Maximum Peak Load. We }}$ assume that the total peak load in California is $26000 \mathrm{MW}$ and the total number of cars in California is $13.3 \times 10^{6}$. This ratio is kept constant to simulate areas of different size and study the impact of PEV penetration in California. Figure 5 shows the impact of PEV penetration on a 3MW peak area, which approximately corresponds to 1600 cars. We consider the only available charging infrastructure is L1 chargers at home. It is remarkable to see that $20 \%$ penetration ( 315 PEVs) suffices to reduce the evening ramping by a factor of 2 .

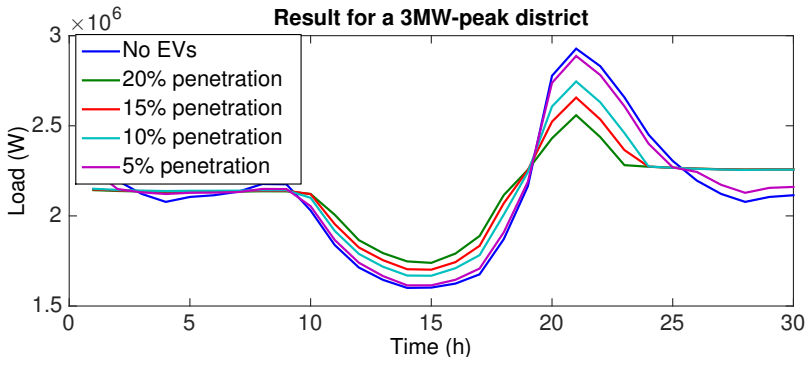

Fig. 5. Impact of EV penetration on Demand Response opportunities

\section{Impact of driver mobility}

In this section, we analyze the effect of driving schedule variability on the results. We use a simultaneous activitybased approach to generate different realistic mobility profiles, based on NHTS data. More particularly, a simultaneous model first deconstructs a person's day into a set of activities, and independently simulates each of them [25]. The driving schedule generation includes two major steps:

- Identify 'tour profiles'. In this first step, we divide the dataset into 20 different day-profiles, which represent typical trip and activity day schedules (for example "Home / Trip 1 / Work / Trip 2 / Home"). We compute the percentage of people that fall into each profile.

- Statistical analysis of activity duration and trip distance. By using distribution inference and parameter fitting, we assess the most representative probabilistic distribution of each schedule element.

Eventually, the NHTS dataset is modeled by 20 different activity profiles, which can be used to simulate any representative population and travel demand [25], [24].

Figures 6 and 7 show the result for different fleets of 200 cars and 180 cars respectively. Figure 6 and 7 s show the number of plugged-in cars during the time period and Fig.6 6 , $7 \mathrm{a}$ and $6 \mathrm{~b}, 7 \mathrm{p}$ show the result of the control algorithm. Figure 6 features a case where fleets have pretty similar driving patterns (mostly commuters). In this case, the price signal slightly varies across fleets. On the contrary Fig. 7 features two different fleets: Fleet 1 is a mix of night and day workers with chargers at work, and Fleet 2 is a population of dayworkers with no charger at work. In this case, the price signal noticeably differs.

This price signal has an intuitive meaning: when the system is over-utilized (evening peak loads), the price signal is high, whereas when the system is under-utilized (day valley), the price signal is low. This pattern is independent from driving behaviors and can be compared to a marginalprice-type of signal [9]. However, contrary to a marginal price, the case study shows that the optimal price signal also depends on driving behaviors (Fig. 7b). This shows that a good forecast of trip schedules will improve the efficiency of the control algorithm.

\section{CONClusion}

This paper describes distributed load shaping strategies to control PEV charging schedules via dual splitting. We define 

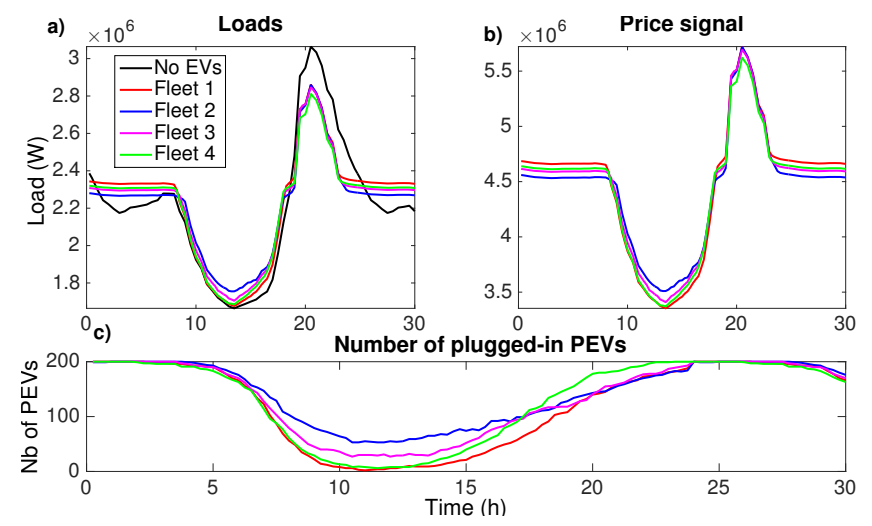

Fig. 6. Sensitivity to driving schedules
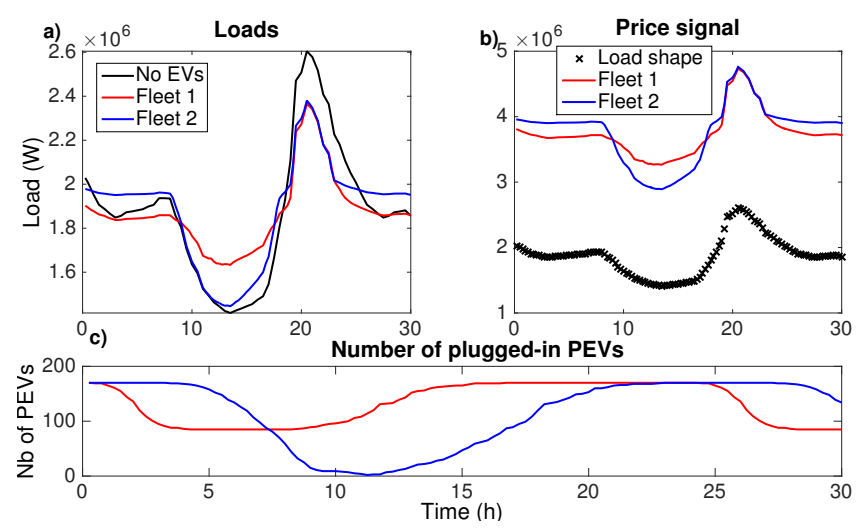

Fig. 7. Impact of driving schedules and charging infrastructure

a global optimization problem, which aims at coordinating PEVs to minimize the load variance. We show that this objective can be distributed and solved in a decentralized framework. In the first step each PEV solves a local optimal program based on a broadcast price signal, and communicates their response to the aggregator. In the second step, the aggregator updates the price signal to reach minimal load variance. We propose three methods to compute this iterative process and prove their characteristics. First, the gradient ascent method converges in a linear rate but needs an update from every agent at each iteration. Second, the Stochastic Incremental Method with constant step-size converges in a linear rate, needs the update from only one agent at each iteration but converges to an approximate solution. Third, the the Stochastic Incremental Method with decreasing step-size converges to the optimal solution as $\frac{1}{k}$ and needs the update from only one agent at each iteration. Finally, we compute several case studies based on real data, and demonstrate that PEVs provide a compelling opportunity to integrate renewable energy sources in the electricity mix.

\section{REFERENCES}

[1] D. B. Richardson, "Electric vehicles and the electric grid:a review of modeling approaches, impacts, and renewable energy integration," Renewable Sustainable Energy Reviews, vol. 19, pp. 247-254, 2013.
[2] W. Kempton and J. Tomić, "Vehicle-to-grid power fundamentals: Calculating capacity and net revenue," Journal of power sources, vol. 144, no. 1, pp. 268-279, 2005.

[3] A. Langton and N. Crisostomo, "Vehicle-grid integration: A vision for zero-emission transportation interconnected throughout california's electricity system," California Public Utilities Commission, Tech. Rep.

[4] D. Callaway and I. Hiskens, "Achieving controllability of electric loads," Proceedings of the IEEE, vol. 99, no. 1, pp. 184-199, 2011.

[5] K. Clement, E. Haesen, and J. Driesen, "Coordinated charging of multiple plug-in hybrid electric vehicles in residential distribution grids," in Power Systems Conference and Exposition, 2009. PSCE '09. IEEE/PES, March 2009, pp. 1-7.

[6] E. Sortomme, M. Hindi, S. MacPherson, and S. Venkata, "Coordinated charging of plug-in hybrid electric vehicles to minimize distribution system losses," IEEE Transactions on Smart Grid, vol. 2, no. 1, pp. 198-205, March 2011.

[7] C. Le Floch, F. Di Meglio, and S. Moura, "Optimal charging of vehicle-to-grid fleets via pde aggregation techniques," American Control Conference $(A C C), 2015$.

[8] L. Gan, U. Topcu, and S. H. Low, "Optimal decentralized protocol for electric vehicle charging," IEEE Transactions on Power Systems, vol. 28, no. 2, pp. 940-951, 2013.

[9] Z. Ma, D. S. Callaway, and I. A. Hiskens, "Decentralized charging control of large populations of plug-in electric vehicles," IEEE Transactions on Control Systems Technology, vol. 21, no. 1, pp. 67-78, 2013.

[10] W. J. Ma, V. Gupta, and U. Topcu, "Distributed charging control of electric vehicles using regret minimization," in 2014 IEEE 53rd Annual Conference on Decision and Control (CDC), Dec 2014, pp. 49174923.

[11] J. Rivera, P. Wolfrum, S. Hirche, C. Goebel, and H.-A. Jacobsen, "Alternating direction method of multipliers for decentralized electric vehicle charging control," in 2013 IEEE 52nd Annual Conference on Decision and Control (CDC), Dec 2013, pp. 6960-6965.

[12] CAISO. What the duck curve tells us about managing a green grid. [Online]. Available: http://www.caiso.com

[13] USDOT-FHWA, "National Household Travel Survey," U.S. Department of Transportation, Tech. Rep., 2009, http://nhts.ornl.gov/index.shtml.

[14] Y. He, B. Venkatesh, and L. Guan, "Optimal scheduling for charging and discharging of electric vehicles," IEEE Transactions on Smart Grid, vol. 3, no. 3, pp. 1095-1105, 2012.

[15] J. Hu, S. You, M. Lind, and J. Ostergaard, "Coordinated charging of electric vehicles for congestion prevention in the distribution grid," IEEE Transactions on Smart Grid, vol. 5, no. 2, pp. 703-711, 2014.

[16] S. J. Moura, J. L. Stein, and H. K. Fathy, "Battery-health conscious power management in plug-in hybrid electric vehicles via electrochemical modeling and stochastic control," IEEE Transactions on Control Systems Technology, vol. 21, no. 3, pp. 679-694, 2013.

[17] S. B. Peterson, J. Apt, and J. Whitacre, "Lithium-ion battery cell degradation resulting from realistic vehicle and vehicle-to-grid utilization," Journal of Power Sources, vol. 195, no. 8, pp. 2385-2392, 2010.

[18] S. Boyd and L. Vandenberghe, Convex optimization. Cambridge university press, 2004.

[19] D. P. Bertsekas, Nonlinear programming.

[20] A. Nemirovski, A. Juditsky, G. Lan, and A. Shapiro, "Robust stochastic approximation approach to stochastic programming," SIAM Journal on Optimization, vol. 19, no. 4, pp. 1574-1609, 2009.

[21] S. Song, K. Chaudhuri, and A. D. Sarwate, "Stochastic gradient descent with differentially private updates," in IEEE Global Conference on Signal and Information Processing, 2013.

[22] F. Belletti, C. Le Floch, S. Moura, and A. Bayen, "Privacy-preserving dual splitting distributed optimization with application to load flattening in california," in 2015 IEEE 54th Annual Conference on Decision and Control (CDC), 2015.

[23] V2g-sim. [Online]. Available: http://v2gsim.lbl.gov

[24] S. Saxena, C. Le Floch, J. MacDonald, and S. Moura, "Quantifying ev battery end-of-life through analysis of travel needs with vehicle powertrain models," Journal of Power Sources, 2015.

[25] J. L. Bowman and M. E. Ben-Akiva, "Activity-based disaggregate travel demand model system with activity schedules," Transportation Research Part A: Policy and Practice, vol. 35, no. 1, pp. 1-28, 2001. 